\title{
DISPERSION MECHANISM OF AGGREGATE PARTICLES IN AIR
}

\author{
Yasuo KOUSAKA, KiKUo OKUYAMA, ATSUYOSHI SHIMIZU \\ AND TETSUO YOSHIDA \\ Department of Chemical Engineering, University of \\ Osaka Prefecture, Sakai 591
}

\begin{abstract}
Possible dispersion mechanisms of aggregate particles in air stream were proposed and their contribution to dispersion was studied by analyzing the dispersion forces. Various typical dispersers were tested to observe the change in size distribution and some of the theoretical analyses were verified. Impact of particles on some obstacles in air stream and acceleration or deceleration of irregular-shaped aggregates were found as a result, to be effective in aggregate dispersion.
\end{abstract}

\section{Introduction}

Several studies of dispersion of aggregate particles in air stream have been reported ${ }^{1,3,5,10,13,14)}$. These previous works, however, are insufficient to understand the controlling dispersion mechanisms in air stream.

The possible mechanisms of mechanical dispersion in an air stream including those proposed by previous researchers ${ }^{1)}$ are discussed from a theoretical point of view in this paper, and then some of the theoretical analyses will be examined in the light of experiments.

Since the analysis in this study aimed at obtaining a macroscopic understanding of dispersion mechanisms of aggregate particles by fluid flow, the precise analysis and the experimental verification for each mechanism are partly left as a future problem.

\section{Theoretical}

1. 1 Stresses within a spherical particle in a viscous flow $^{1)}$

Bagster and Tomi analyzed the stresses within a spherical particle in a fluid flow for two cases: the stresses arising from a simple shear field and those induced by uniform flow. The maximum stresses of each case, which are important in analyzing the dispersion mechanism, are summarized as follows:

a) simple shear field

$$
\begin{array}{ll}
\sigma_{\gamma 1 \max }=5 \mu \gamma & \text { for tension } \\
\tau_{\gamma \text { max }}=8.5 \mu \gamma & \text { for shear }
\end{array}
$$

\section{b) uniform flow}

Received June 20, 1978. Correspondence concerning this article should be addressed to Y. Kousaka. T. Yoshida is now with Chubu Institute of Technology, Kasugai, Aichi 1200 and A. Shimizu with Chisso Co., Ltd., Chiba 290.

$$
\sigma_{\alpha 1 \max } \text { and } \tau_{\alpha 1 \max }=3 \mu u_{r} / d_{p s}
$$

The maximum stresses expressed above are induced at the instant when a spherical particle is just put into the fluid flow, that is, the particle is just at rest. 1. 2 Stress induced by impact and probability of impact of aggregate particle to obstacle in a fluid flow

If there are obstacles in a fluid flow, particles will collide with them by inertia to induce an impact force within the particles. The impact force is given as follows when a particle collides with an obstacle at a velocity of $v_{i}$,

$$
F_{i}=m v_{i} / \Delta t
$$

where $\Delta t$ represents the duration of impact. The duration of impact for small aggregate particles is expected to be extremely small, judging from experiment $^{6)}$ and the theory of elastic spheres ${ }^{9}$.

The compressive stress at the plane through the particle center induced by impact is expressed as follows, assuming the particle to be spherical.

$$
\sigma_{i}=\frac{2}{3} \rho_{p} d_{p a g}\left(\frac{v_{i}}{\Delta t}\right)
$$

Much higher stress than this value is actually induced at the portion of smaller sectional area of the particle and is also induced by bending moment when the shape of aggregates is irregular. If the stress given by Eq. (5) is assumed to be much larger than the strength of the aggregates because of a sufficiently large value of $v_{i} / \Delta t$, the breakage of the particle depends upon the probability of its impact with the obstable, which is given as

$$
\eta_{T}=f(\phi) \text { where } \phi=\frac{\rho_{p} u_{r} d_{p a g}^{2}}{18 \mu D_{s}}
$$

Suppose a certain value of $\psi_{c}$ when the target efficiency becomes 0.5 . The particle diameter, $d_{p \eta 50}$, corresponding to $\psi_{c}$ is then given as follows: 


$$
d_{p \eta 50}=\sqrt{\frac{18 \mu D_{s} \psi_{s}}{\rho_{p} u_{r}}}
$$

If the particles, half of which impact on an obstacle at the disperser, are observed experimentally, the diameter of such particle will change according to this equation.

\section{3 Stresses within an aggregate particle in accelera-} tion

To simplify the analysis, the model aggregate shown in Fig. 1 is discussed. The analysis of such a particle will give us basic and useful information on aggregate particles of more complicated shape, since such a particle is the elementary unit composing an aggregate. The stress at the contacting point of $\mathrm{A}$ and $B$ particles shown in Fig. 1 is analyzed when this model aggregate is led into a uniform fluid flow oriented as shown in Figs. 1 (a) and (b).

The equation of motion of the model aggregate is given as follows, when A and B particles are assumed to have the same velocity: this assumption is valid for the case of Fig. 1 (b), and is valid at the initiation of acceleration, or at the stationary state of the particle just being put into a stream, for the case of Fig. 1 (a).

$$
\begin{array}{ll}
m_{A}\left(d u_{p} / d t\right)=R_{f A}-F & \text { on the basis of particle A } \\
m_{B}\left(d u_{p} / d t\right)=R_{f B}+F & \text { on the basis of particle B }
\end{array}
$$

Stokes' law and the following relations are assumed here

$$
\begin{aligned}
& R_{f A}=3 \pi \mu u_{r} d_{p A} \\
& R_{f B}=3 \pi \mu u_{r} d_{p B}
\end{aligned}
$$

Assuming $\rho_{A}=\rho_{B}=\rho_{p}$, Eqs. (8) and (9) reduce to the next equation.

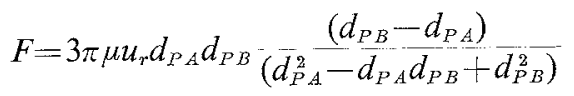

This gives the force acting on the contacting area between $\mathrm{A}$ and $\mathrm{B}$ particles, defined as

$$
A_{r}=\frac{\pi}{4}\left(c_{1} d_{P A}\right)^{2}
$$

The value of $c_{1}$ will depend upon the manner of adhesion of $\mathrm{A}$ and $\mathrm{B}$ particles, and is expected to have a certain distribution. The shearing stress and the tensile stress at the contact point are given, for the cases of Figs. 1 (a) and (b) respectively, as

$$
\tau_{\alpha^{2}} \text { and } \sigma_{\alpha 2}=4 F / \pi\left(c_{1} d_{P A}\right)^{2}
$$

The bending stress for the case of Fig. 1 (a) is given as follows.

$$
\sigma_{\alpha 3}=\frac{(1 / 2) d_{P A} F}{(\pi / 32)\left(c_{1} d_{P A}\right)^{3}}=16 F / \pi c_{1}\left(c_{1} d_{P A}\right)^{2}
$$

Since $c_{1}$ is less than unity, $\sigma_{\alpha 3}$ is larger than $\tau_{\alpha 2}$ and $\sigma_{\alpha 2}$. This means that a model aggregate having the orientation shown in Fig. 1 (a) is more easily disintegrated. Substituting Eq. (12) into Eqs. (14) and (15) and solv-

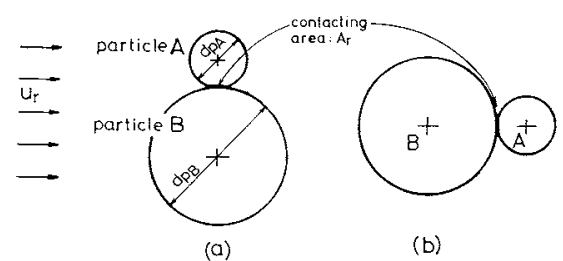

Fig. 1 Illustration of a model aggregate

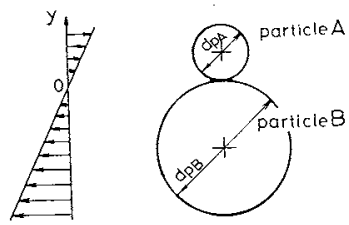

Fig. 2 Illustration of a model aggregate put in simple shear field

ing it as to $d_{P B}$, it follows that:

for the case of Fig. 1 (b):

$$
d_{P B}=\frac{d_{P A}}{2}\left(1+\frac{\sqrt{3} \sqrt{48} \lambda_{1}^{2}+8 \lambda_{1}-1}{12 \lambda_{1}-1}\right)
$$

where

$$
\lambda_{1}=-\frac{\mu u_{r}}{c_{1}^{2} \sigma_{\alpha 2}} d_{P A}
$$

for the case of Fig. 1 (a):

$$
d_{P^{B}}=\frac{d_{P}}{2}\left(1+\frac{\sqrt{3} \sqrt{7 \overline{6} 8 \overline{\lambda_{2}^{2}}+\overline{3} \overline{\lambda_{2}}-1}}{48 \overline{\lambda_{2}-1}}\right)
$$

where

$$
\lambda_{2}=\frac{\mu u_{r} .}{c_{1}^{3} \sigma_{\alpha 3}} d_{P A}
$$

If $\sigma_{\alpha 2}$ and $\sigma_{\alpha 3}$ are replaced by $\sigma_{p}$, the strength of the aggregate particle, the stable size of $\mathrm{B}$ particle is determined by Eqs. (16) and (18). The dimensionless parameters $\lambda_{1}$ and $\lambda_{2}$ have the meaning of the ratio of breaking force to the strength of the contact point. From Eqs. (16) and (18) it is seen that A and B particles are more difficult to be separated when their sizes become similar and that the distintegration of small aggregates is much easier than that of large aggregates.

To compare this analysis with Eq. (3), the following stresses, $\tau_{\alpha 3}^{\prime}, \sigma_{\alpha 2}^{\prime}$ and $\sigma_{\alpha 3}^{\prime}$ at the condition of $d_{p B}=$ $1.2 d_{P_{A}}$, are now considered as an example.

$\tau_{\alpha 2}^{\prime}$ and $\sigma_{\alpha 2}^{\prime} \simeq \frac{3 \mu u_{r}}{c_{1}^{2} d_{P B}} \quad$ for the case of Fig. 1 (b)

$\sigma_{\alpha 3}^{\prime} \simeq \begin{gathered}12 \mu u_{r} \\ c_{1}^{3} \bar{d}_{P B}\end{gathered}$ for the case of Fig. 1 (a)

It should be emphasized that the above stresses are extremely large compared with that of a spherical particle.

\section{4 Stress within an aggregate particle in simple shear field}

Figure 2 illustrates the case to be analyzed in this section. The maximum stress in this case is caused 


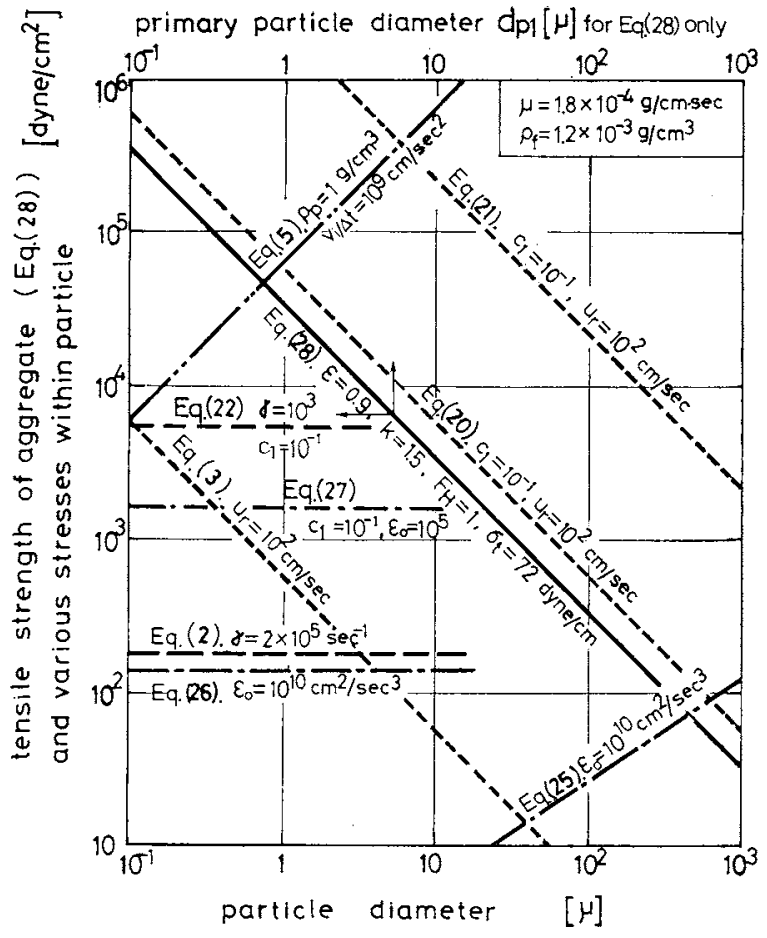

Fig. 3 Comparison among various stresses and strength of powder bed

when the particle is at rest or just put into the flow. To estimate the order of magnitude of the bending stress which is much larger than the shearing stress, $B$ particle is assumed to be sufficiently larger than A particle. Then it follows that

$$
\begin{gathered}
\sigma_{\gamma 2}=\frac{\int_{0}^{d_{P A}} y d R_{f}}{(\pi / 32)\left(c_{1} d_{P A}\right)^{3}} \approx \frac{93 \mu \gamma}{\pi c_{1}^{3}} \\
\frac{\sigma_{\alpha 3}^{\prime}}{\sigma_{\gamma 2}} \simeq 0.4 \times \frac{1}{\gamma}\left(\frac{u_{r}}{d_{P B}}\right) \\
\frac{u_{r}}{d_{P B}}<2.5 \gamma \text { for } \sigma_{\alpha 3}^{\prime}>\sigma_{\gamma 2}
\end{gathered}
$$

Equation (24) is most likely valid for the usual case where the dispersion of small particles is discussed. This means that the effect of the component of velocity gradient on the stress within a small aggregate particle is less important than that of acceleration in uniform flow. The stress given by Eq. (22), however, is much larger than those given by Eqs. (1) and (2) for a spherical particle.

The particle shown in Fig. 2 will rotate with the lapse of time, decreasing the stress given by Eq. (22), and instead causing a stress induced by centrifugal force which is difficult to evaluate, but is expected not to be significant from the analysis for a spherical pariticle ${ }^{11}$.

\subsection{Stresses within a spherical particle in turbulent flow}

The analysis discussed in section 1.3 are essen- tially applicable to turbulent flow.

It is difficult, however, to evaluate the local relative velocity in turbulent flow. Thus the stresses within a spherical particle induced by velocity gradient in a locally isotropic turbulent flow are discussed here.

According to Kolmogoroff's theory the average mean-square velocity between two points is given for the former case by $\tilde{u}_{r}^{2} \simeq\left(\varepsilon_{0} r\right)^{2 / 3}$. The shearing stress at the plane through the center of a spherical particle induced by the inertial force of fluid may be roughly estimated as

$$
\tau_{\varepsilon 1} \simeq \frac{\rho_{f} \bar{u}_{r}^{2}}{2} \cdot \frac{\pi d_{p s}^{2}}{8} \cdot \frac{4}{\pi d_{P S}^{2}} \simeq \frac{\rho_{f} \varepsilon_{0}^{2 / 3}}{10} d_{P S}^{2 / 3} \quad r \gg \lambda_{s}
$$

When the particle size is smaller than the microscale and the viscous shear force becomes important, the shearing stress is given using the relation ${ }^{8)}$ of $(\partial \overline{u / \partial r})^{2} \simeq 2 \varepsilon_{0} / 15 \nu$ and Eq. (2), as

$$
\tau_{\mathrm{e} 2} \simeq 3.1 \rho_{f} \sqrt{\nu \varepsilon_{0}} \quad r \ll \lambda_{s}
$$

If Eq. (22) is applied instead of Eq. (2) for the case of $r \ll \lambda_{s}$, the bending stress is given as

$$
\sigma_{\varepsilon 3} \simeq \frac{10.8 \rho_{f} \sqrt{\nu \varepsilon_{0}}}{c_{1}^{3}} \quad r \ll \lambda_{s}
$$

\section{6 Comparison among the stresses}

Figure 3 collectively illustrates the stresses above analyzed. The solid line in the figure shows the strength of a powder bed resulting from water bridge $^{7)}$, which is given as

$$
\sigma_{p}=\frac{1-\varepsilon}{\pi d_{p 1}} k F_{I I} \sigma_{t}
$$

As far as small spherical particles are concerned, the stresses induced by inertial force and viscous force of a fluid in a turbulent flow, Eqs. (25) and (26), and the stress induced by viscous force in simple shear field, Eqs. (2), seem to be very small in spite of the unusually large values of $\varepsilon_{0}$ and $\gamma$ assumed in the figure. Only one mechanism of dispersion of small spherical particles in an air stream is conclusively said to be that by impact of the particle on obstacles in the stream.

The effective mechanisms of dispersion of small non-spherical particles, on the other hand, are those by impact, acceleration and by velocity gradient. But the last one is not so significant compared with the other two mechanisms.

The results of the analyses in this paper may be essentially applied to dispersion of aggregates in liquid excluding electrical effect, and it will be found that acceleration of aggregates plays an important role in dispersion in liquid while impact has less effect in liquid than it has in air.

\section{Experimental}

\section{1 Experimental apparatus}


Figure 4 shows the various dispersers to be tested. Figure 4 (a) is a disperser to verify the dispersion mechanism by impact of aggregate particles on fine wires inside the pipe. If the dispersion of aggregates takes place in this disperser, existence of dispersion mechanism by impact will be verified. Figure 4 (b) is a disperser to examine the dispersion mechanism by acceleration or deceleration of aggregates. Two kinds of venturi having different converging and diverging angles are prepared to check the effect of the magnitude of acceleration. The dispersion mechanisms at the orifice shown in Fig. 4 (c) are complicated. The possible mechanisms will be those by acceleration of particles due to sudden contraction of air stream, by impact of particle at the orifice edge, by velocity gradient of stream and those by turbulence. The difference between the orifice and the capillary which is shown in Fig. 4 (d) exists only in the length of contraction zone. Since Figs. 4 (b), (c) and (d) have the same dimensions in inlet diameter and in contraction diameter, it is interesting to see the difference of experimental results among them. The rotating-blade disperser shown in Fig. 4 (e) is also thought to have composite dispersion mechanism and seems to be inappropriate for a fundamental study.

A schematic diagram of the experimental apparatus is shown in Fig. 5. Aggregate particles were generated from Chujo's container ${ }^{4}$ and were dispersed in a short time into a reservoir having the capacity of 36 liters. Then the aggregate particles in suspension in the reservoir were led into the dispersion section, making up the fresh air from the Chujo's container, where one of the various dispersers shown in Fig. 4 was installed. Particle size of aggregates after the dispersers was analyzed by means of an ultramicroscopic technique based upon airy sedimentation which was previously developed by the authors ${ }^{12)}$. The powder materials used in this study were $\mathrm{CaCO}_{3}$ (geometric mean diameter of primary particles $d_{p 1}=$ $0.64 \mu$, density $\left.\rho_{p 1}=2.6 \mathrm{~g} / \mathrm{cm}^{3}\right)$ and $\mathrm{Fe}_{2} \mathrm{O}_{3}\left(d_{p 1}=0.31\right.$ $\left.\mu, \rho_{p 1}=5.2 \mathrm{~g} / \mathrm{cm}^{3}\right)$. The size distributions of primary particles obtained by sedimentation in water are shown in Fig. 6. The size distributions in Fig. 6 and those in the following figures are all plotted on a number basis. The moisture content of the powders were $0.12-0.15 \%$ for $\mathrm{CaCO}_{3}$ and $0.28-0.43 \%$ for $\mathrm{Fe}_{2} \mathrm{O}_{3}$. The bulk densities of aggregates were assumed to be those of the powder beds at maximum porosity, which were $0.88 \mathrm{~g} / \mathrm{cm}^{3}$ for $\mathrm{CaCO}_{3}$ and 0.71 $\mathrm{g} / \mathrm{cm}^{3}$ for $\mathrm{Fe}_{2} \mathrm{O}_{3}$. Particle number concentration of aggregate particles in suspension was less than $10^{6}$ particle $/ \mathrm{cm}^{3}$, which has little effect on simultaneous coagulation. The humidity of air in the reservoir was from $60 \%$ to $80 \%$, within which range little effect on the experimental result was found.

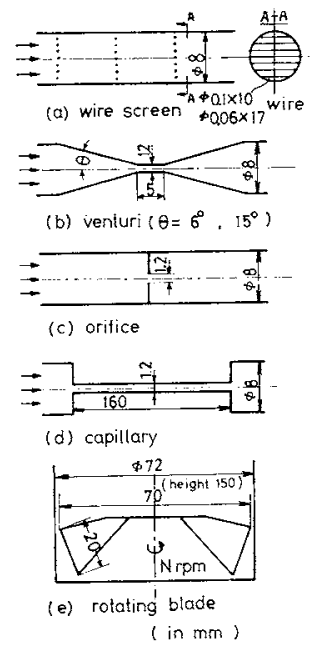

Fig. 4 Dispersers used in experiment

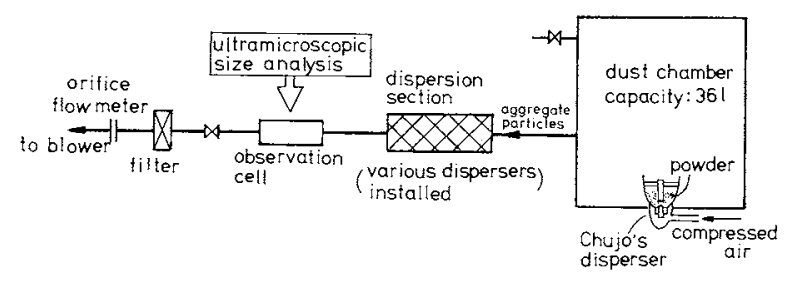

Fig. 5 Arrangement of experimental apparatus

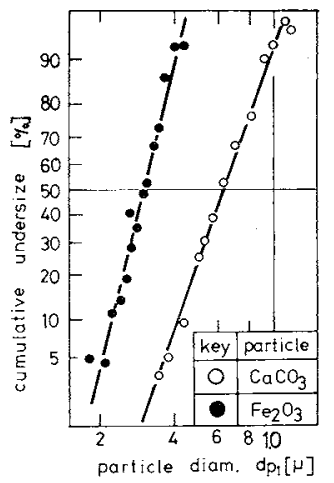

Fig. 6 Size distribution of primary particles

\section{2 Experimental results and discussion}

1) Dispersion by impact In the theoretical analysis it was suggested that one of the most important mechanisms of dispersion is that by impact of particles upon obstacles in an air stream. Figure 7, for example, shows the size distribution change with air velocities in a pipe having 8-stage wire screens. Because of the high target efficiency of larger particles to the wire screens, the fraction of larger particles decreases with increase of air velocity. It is also understood from Fig. 8 that the impact of large particles on the wire screens results in generating smaller particles with high number concentration. Figure 9 shows the effect of air velocity on the various aggregate sizes, $d_{p 50}, d_{p 90}$ and $d_{p \eta 50}$, after dispersion. It is seen in the figure that the effect of 

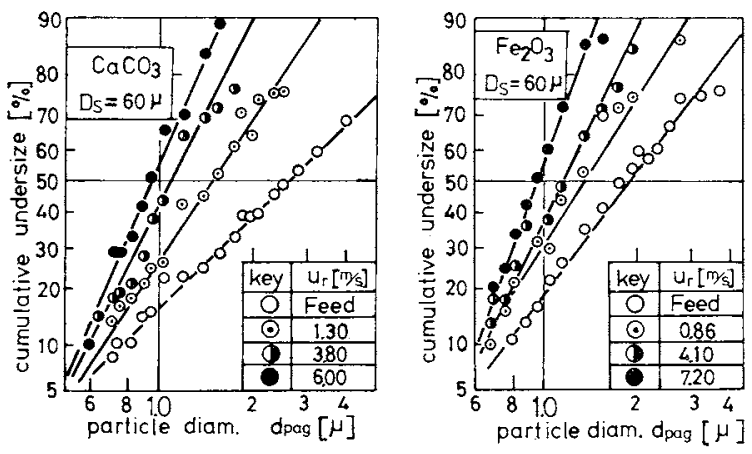

Fig. 7 Change in size distributions of aggregates obtained by the disperser shown in Fig. 4 (a)

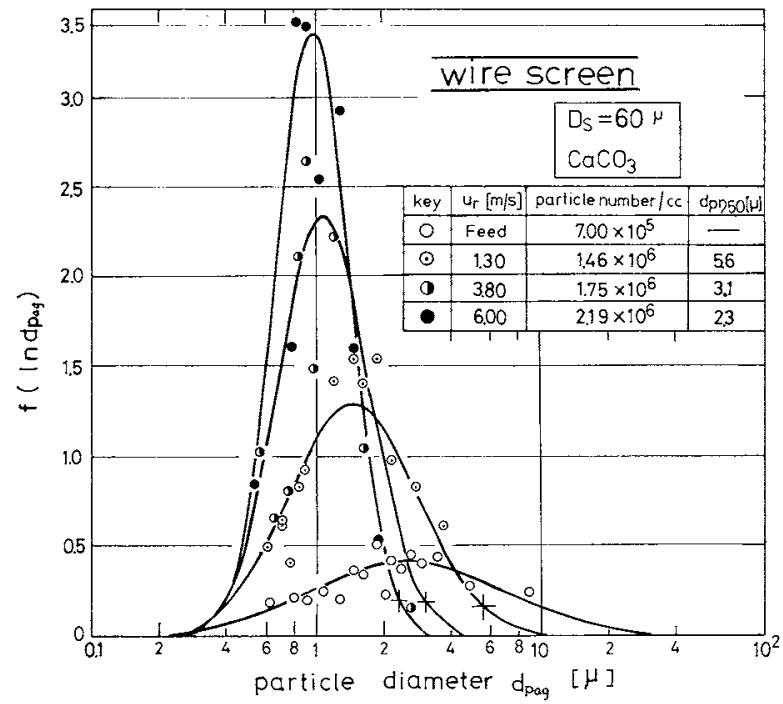

Fig. 8 Change in number and size distributions of aggregates obtained by the disperser shown in Fig. 4 (a)

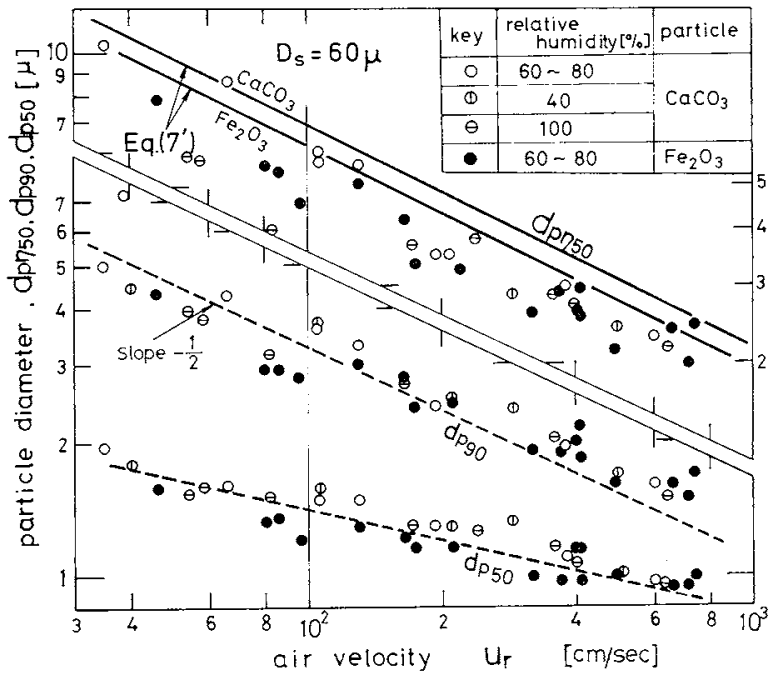

Fig. 9 Size change of aggregates dispersed by wire screens

relative humidity of dispersion air is not serious under the conditions of relative humidities above $40 \%$. The diameters, $d_{p 50}$ and $d_{p 90}$, in Fig. 9 are for comparison with those obtained by the other dis-

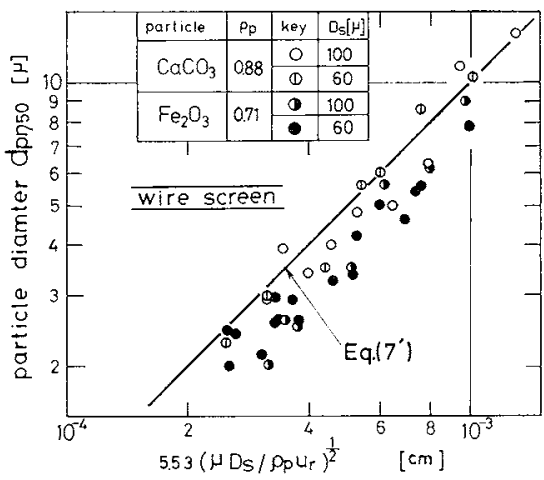

Fig. 10 Comparison of Eq. $\left(7^{\prime}\right)$ with experimental results obtained by the disperser shown in Fig. 4 (a)

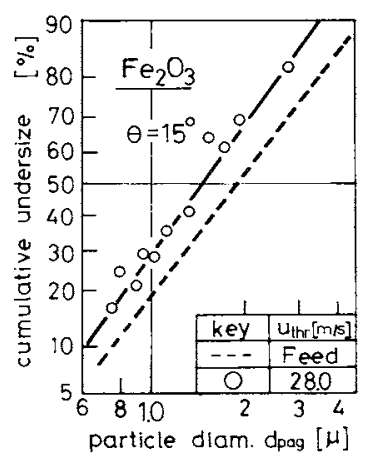

(a)

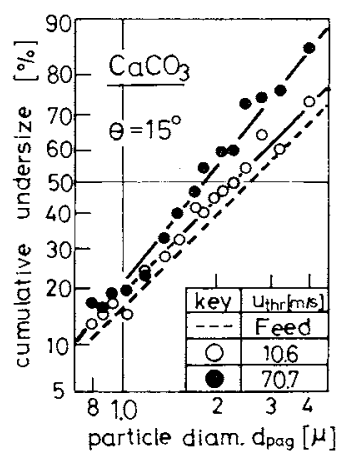

(b)
Fig. 11 Change in size distributions of aggregates obtained by the disperser shown in Fig. 4 (b)

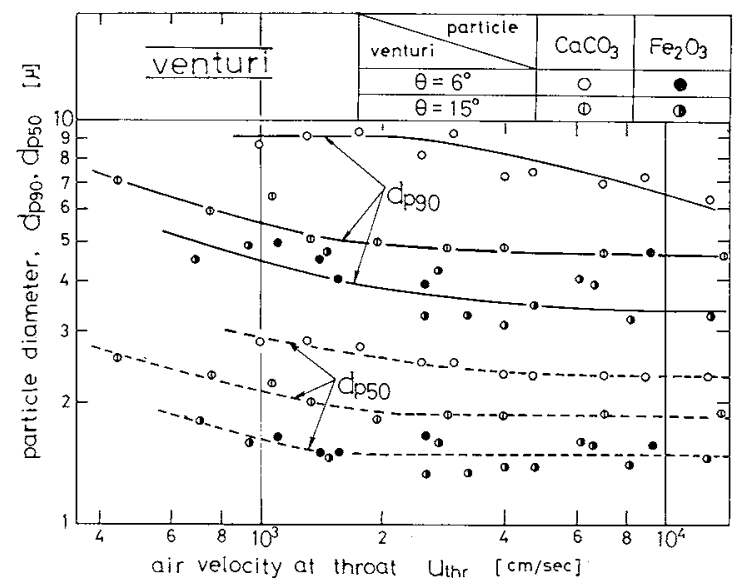

Fig. 12 Size change of aggregates dispersed by venturi

persers, which appear in the later sections. The diameter, $d_{p \eta 50}$, is defined as that corresponding to causing of $50 \%$ number decrease, shown in Fig. 8 as cross marks. The corresponding theoretical diameter, $d_{p \eta 50}$, is determined as follows. Particles larger than $d_{p \eta 50}$ which collide with the obstacle are assumed to fall apart into smaller particles than $d_{p \eta 50}$. The overall target efficiency in this experiment can be given assuming complete mixing after each stage as follows.

$$
\eta_{T}=1-\left[1-(1-\varepsilon) \eta_{T_{1}}\right]^{N_{s}}
$$




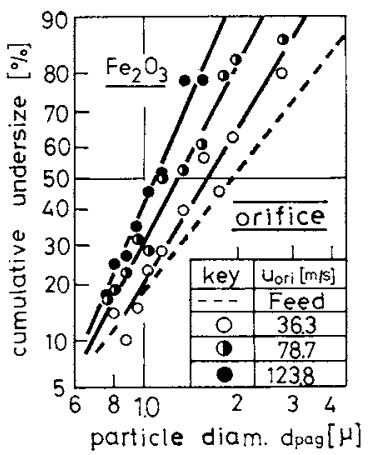

(a)

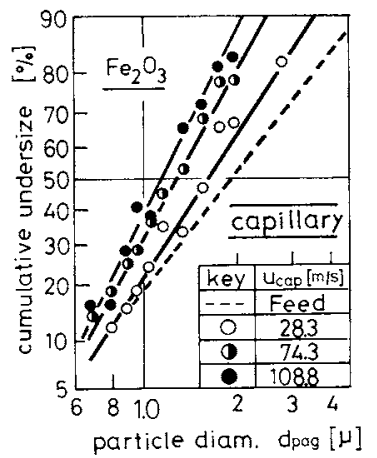

(b)

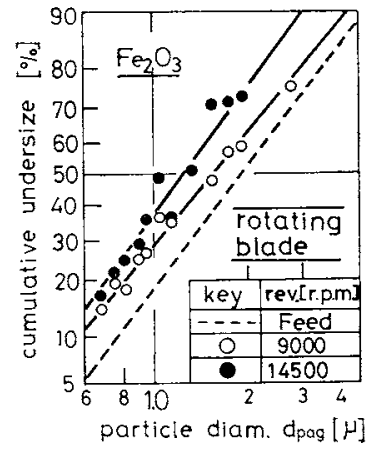

(c)
Fig. 13 Comparison among the size distribution changes obtained by the dispersers shown in Figs. 4 (c), (d) and (e)
Substituting $\eta_{T}=0.5, \varepsilon=0.125$ and $N_{s}=8$ into this equation, $\eta_{T_{1}}$ becomes 0.66 . The value of $\psi_{c}$ at $\eta_{T_{1} 1}=0.66$ is given as 1.7 using Davies' result ${ }^{2)}$ for an isolated cylinder at $R e=10$ without interception effect. Then Eq. (7) becomes

$$
d_{P \eta 50}=5.53 \sqrt{\frac{\mu D_{s}}{\rho_{p} u_{r}}}
$$

The solid lines in Fig. 9 show this relation. Figure 10 shows the correlation of all of the experimental results and Eq. $\left(7^{\prime}\right)$. The satisfactory agreement between Eq. $\left(7^{\prime}\right)$ and the experimental results implies that dispersion of aggregate particles by impact is effective and that at the same time the stress induced by impact is sufficiently larger than the strength of aggregate particles.

2) Dispersion by acceleration Figure $\mathbf{1 1}$ shows the size distribution change obtained by the venturi shown in Fig. 4 (b). Differing from Fig. 7, a slight change in the width of size distribution is found after dispersion. Dispersion by acceleration is effective for small aggregates as suggested from Eqs. (20) and (21) if the relative velocity $u_{r}$ in the equations is independent of particle size. The relative velocity, however, actually decreases in the venturi with decrease in particle size. As a result of these two effects, the size distributions will not be spread nor sharpened, as seen in Fig. 11.

Figure 12 shows the size change of $d_{p 50}$ and $d_{p 90}$ with venturi throat velocity. The effect of the size of feed particles appears, which is expected from the analysis and is different from the case of impact dispersion. It is a matter of course that particle size decreases with increase of convergent angle $\theta$. Because of the difficulty in obtaining a large relative velocity of a particle to air in the venturi experiment, dispersion by acceleration seems to be less effective than that by impact using wire screens.

3) Experimental results obtained by the dispersers shown in Figs. 4 (c), (d) and (e) Figure 13 shows the comparison of the size distribution change after dispersion for three dispersers. Figure 13 (a) is very

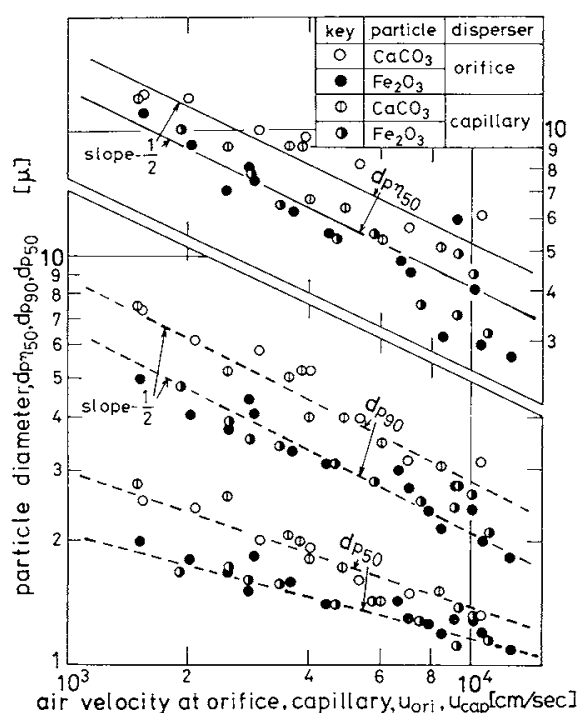

Fig. 14 Size change of aggregates dispersed by orifice and capillary

similar to Fig. 13 (b), which suggests that the controlling dispersion mechanism of both orifice and capillary shown in Figs. 13 (c) and (d) is almost the same. Further, Figs. 13 (a) and (b) are similar to Fig. 7, and thus the controlling dispersion mechanism of these dispersers seems to be impact. Figure 14 shows the size change with mean air velocity at the contraction section of the dispersers. The tendency of these results is similar to that shown in Fig. 9, except for the considerable size difference between $\mathrm{CaCO}_{3}$ and $\mathrm{Fe}_{2} \mathrm{O}_{3}$ aggregates. This size difference is caused by the effect of acceleration and deceleration at the contraction zone of these dispersers in addition to the impact effect. Because the orifice and the capillary have the same dimension at their contraction zone, aggregates collide with the inlet plate of the capillary as well as the orifice plate by inertia in the same way as with the wire screens. No available target efficiency analyzed for the orifice flow has been reported so far, and no quantitative discussion can be made for these cases. However, the fact that the diameter $d_{p \eta 50}$ is inversely propor- 


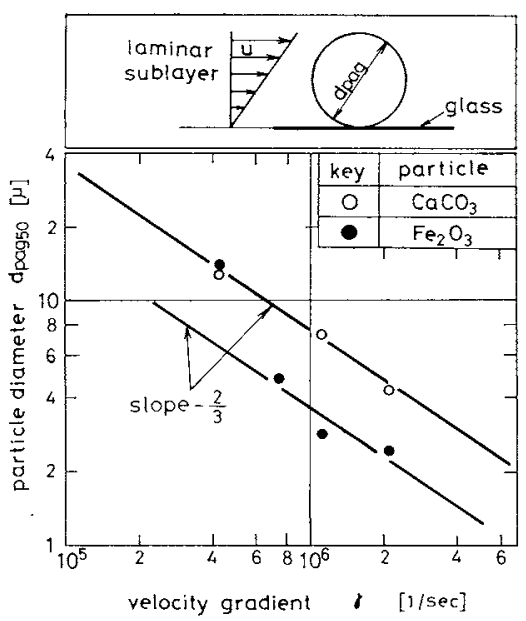

Fig. 15 Experimental result of re-entrainmentrelation between the size such that $50 \%$ of aggregate particles is re-entrained and velocity gradient-

tional to the square root of the air velocities, $U_{\text {ori }}$ and $U_{c a p}$, is enough to prove impact dispersion, since the $d_{p \eta 50}$ in these cases should be expressed in the same form as Eq. (7).

Though the length of the capillary tube was changed from $6 \mathrm{~cm}$ to $50 \mathrm{~cm}$, no effect of length on size change was found, a fact also reported by Yamamoto ${ }^{11}$. This suggests that the velocity gradient in the tube has little effect on dispersion, which is also expected from the analysis in section 1.4. The small effect of the velocity gradient is also suggested from another experiment, shown in Fig. 15. The particle diameter $d_{p a g 50}$ in the figure is the aggregate size such that $50 \%$ of the particles is re-entrained by the air stream. It is seen that the small deposited particles on the glass wall cannot be re-entrained by the air stream, in spite of the large velocity gradient in the laminar sublayer established just above the wall.

Figure 13 (c) is the result obtained by the rotatingblade disperser shown in Fig. 4 (e). The size change is obviously different from those of the other two figures. This figure is similar to Fig. 11 obtained by a venturi, and thus the controlling dispersion mechanism of the rotating-blade disperser seems to be acceleration. The dispersion by this experiment, where the aggregate particles are fed into the disperser as an aerosol, seems to be less effective in spite of violent turbulence than that by the other two dispersers. The same disperser, however, can be fairly effective for dispersion when aggregates are fed directly into the disperser not as an aerosol but as agglomerates. The impact action at the blade will improve dispersion because the aggregates powder are sufficiently large to be hit by the blade in this case.

\section{Conclusion}

The following results were obtained from both theoretical and experimental analyses:

1) Dispersion of aggregate particles caused by their impact on obstacles in an air stream is very effective, and size reduction by impact can be estimated from the existing theory of the target efficiency of a particle in a stream.

2) Dispersion of an aggregate particle caused by acceleration or deceleration, including that in turbulence, is fairly effective when the relative velocity of the particle to fluid is large. The shape of a particle has an important role in this case; a spherical particle is difficult to disperse while an irregular-shaped aggregare is much easier to disperse.

3) Dispersion of an aggregate particle caused by velocity gradient in a fluid flow is less effective than those mentioned above, even if particle shape is taken into account.

\section{Nomenclature}

$A_{r} \quad=$ contacting area of $\mathrm{A}$ and $\mathrm{B}$ particles shown

in Fig. 1 [ $\left.\mathrm{cm}^{2}\right]$

$\begin{array}{lll}c_{1} & =\text { coefficient defined as Eq. (13) } & {[-]} \\ D_{s} & =\text { diameter of an obstacle } & {[\mathrm{cm}],[\mu]}\end{array}$

$d_{p} \quad=$ particle diameter $[\mathrm{cm}, \mu]$

$\begin{array}{lll}d_{p 1} & =\text { primary particle diameter } \quad[\mathrm{cm}, \mu]\end{array}$

$d_{p a g} \quad=$ diameter of an aggregate particle $\quad[\mathrm{cm}, \mu]$

$d_{p s} \quad=$ diameter of a spherical particle $\quad[\mathrm{cm}, \mu]$

$d_{p \eta 50}=$ particle diameter such that half of the particles collides with an obstacle [cm, $\mu]$

$d_{p 50}, d_{p 90}=$ particle diameter of $50 \%$ and $90 \%$ cumulative undersize, respectively [cm, $\mu]$

$F \quad=$ force acting on contacting area of $A$ and $B$ particles

$F_{H} \quad=$ dimensionless adhesive force

$F_{i} \quad=$ impact force

$k \quad=$ coordination number

$m \quad=$ mass of particle

$N_{s} \quad=$ number of stages of wire screen

$R_{f} \quad=$ drag force

$r \quad=$ distance

t $\quad=$ time

$\Delta t \quad=$ duration of impact

$u \quad=$ fluid velocity

$u_{c a p} \quad=$ mean air velocity in capillary

$u_{o r i} \quad=$ mean air velocity at orifice

[dyne]

[-]

[dyne]

$[-]$

[g]

$[-]$

[dyne]

$[\mathrm{cm}]$

[sec]

[sec]

$[\mathrm{cm} / \mathrm{sec}]$

$[\mathrm{cm} / \mathrm{sec}]$

$[\mathrm{cm} / \mathrm{sec}]$

$u_{p}, u_{p A}, u_{p B}=$ particle velocities of model aggregate, $A$, and $B$ particles, respectively

[cm/sec]

$u_{r} \quad=$ mean air velocity in a pipe or relative velocity shown in Fig. $1 \quad$ [cm/sec]

$=$ mean air velocity at throat

$\begin{array}{lll}v_{i} & =\text { impact velocity } & {[\mathrm{cm} / \mathrm{sec}]}\end{array}$

$y=$ distance shown in Fig. $2 \quad[\mathrm{~cm}]$

$[\mathrm{cm} / \mathrm{sec}]$

$\gamma \quad=$ velocity gradient $\quad[1 / \mathrm{sec}]$

$\varepsilon \quad=$ porosity [-]

$\varepsilon_{0} \quad=$ average energy dissipation rate $\quad\left[\mathrm{cm}^{2} / \mathrm{sec}^{3}\right]$

$\eta_{T} \quad=$ target efficiency [-]

$\eta_{T 1} \quad=$ target efficiency for single stage $\quad[-]$

$\lambda_{1}, \lambda_{2}=$ dimensionless parameter defined as Eqs. (17) and (19)

$\lambda_{s}=$ turbulent micro-scale $\quad[\mathrm{cm}, \mu]$

$\mu \quad=$ viscosity $\quad[\mathrm{g} / \mathrm{cm} \cdot \mathrm{sec}]$ 
$\begin{array}{llr}\nu & =\text { kinematic viscosity } & {\left[\mathrm{cm}^{2} / \mathrm{sec}\right]} \\ \rho_{f} & =\text { density of fluid } & {\left[\mathrm{g} / \mathrm{cm}^{3}\right]} \\ \rho, \rho_{p} & =\text { particle density } & {\left[\mathrm{g} / \mathrm{cm}^{3}\right]} \\ \rho_{p 1} & =\text { density of primary particle } & {\left[\mathrm{g} / \mathrm{cm}^{3}\right]} \\ \sigma_{i} & =\text { stress induced by impact } & {\left[\mathrm{dyne} / \mathrm{cm}^{2}\right]} \\ \sigma_{\alpha 1 \mathrm{max}}, \sigma_{\gamma 1 \mathrm{max}} & =\text { tensile stresses within a sphere analyzed } \\ & \text { by Bagster, Eqs. (3) and (1), respectively }\end{array}$

by Bagster, Eqs. (3) and (1), respectively
[dyne $\left./ \mathrm{cm}^{2}\right]$

$\sigma_{\alpha 2}, \sigma_{\alpha 3}=$ tensile and bending stress at contacting area of model aggregate caused by uniform flow [dyne $/ \mathrm{cm}^{2}$ ]

$\sigma_{p} \quad=$ strength of powder bed $\quad\left[\mathrm{dyne} / \mathrm{cm}^{2}\right]$

$\begin{array}{lll}\sigma_{t} & =\text { surface tension } & \text { [dyne } / \mathrm{cm}]\end{array}$

$\sigma_{\alpha 2}^{\prime}, \sigma_{\alpha 3}^{\prime} \quad=\sigma_{\alpha 2}$ and $\sigma_{\alpha 3}$ at $d_{p B}=1.2 d_{p A} \quad$ [dyne $\left./ \mathrm{cm}^{2}\right]$

$\sigma_{\gamma 2}, \sigma_{\varepsilon 3}=$ bending stress at contacting area of model aggregate caused by simple shear flow, and caused by velocity gradient in turbulent flow, respectively $\quad\left[\mathrm{dyne} / \mathrm{cm}^{2}\right]$ $\tau_{\alpha 1 \mathrm{max}}, \tau_{\gamma 1 \mathrm{max}}=$ shearing stresses within a sphere analyzed by Bagster, Eqs. (3) and (2), respectively

[dyne $/ \mathrm{cm}^{2}$ ]

$\tau_{\alpha 2}=$ shearing stress at contacting area of model aggregate

[dyne $\left./ \mathrm{cm}^{2}\right]$

$\tau_{\alpha 2}^{\prime} \quad=\tau_{\alpha 2}$ at $d_{p B}=1.2 d_{p A} \quad$ [dyne $/ \mathrm{cm}^{2}$ ]

$\tau_{\varepsilon 1}, \tau_{\varepsilon 2}=$ shearing stress within a sphere inđuced by inertial force of fluid and velocity gradient, respectively, in turbulent flow

[dyne $/ \mathrm{cm}^{2}$ ]

$\phi \quad=$ inertial parameter

$\psi_{c} \quad=$ inertial parameter at $\eta_{T}=0.5$ $\langle$ Subscripts〉

$\mathrm{A}, \mathrm{B}=$ particles $\mathbf{A}$ and $\mathbf{B}$ shown in Fig. 1

Literature Cited

1) Bagster, O. F. and D. Tomi: Chiem. Eng. Sci., 29, 1773 (1974).

2) Davies, C. N. and C. V. Peetz: Proc. Roy. Soc., A234, 269 (1956).

3) Denice, R.: "Handbook on Aerosols", p. 20, Tech. Inf. Center, U.S. ERDA (1976).

4) Iinoya, K. ed.: "Funtai Kogaku Handbook", Asakura Shoten (1965).

5) Masuda, H., S. Fushiro and K. Iinoya: J. of Assoc. Powder Technology, Japan, 14, 3 (1977).

6) Nakagawa, Y., S. Okuda S. Inamura and A. Yashima: Kagaku Kögaku, 22, 624 (1958).

7) Rumpf, H.: Chem. Ing. Tech., 42, 538 (1970).

8) Shinnar, R.: J. Fluid Mech., 10, 259 (1961).

9) Timoshenko, S.: "Theory of Elasticity", (2nd ed.) p. 384, Marple Press (1951).

10) Tsubaki, J. and H. Hirano: 37th Kenkyu Danwakai, 75 (1977).

11) Yamamoto, H., A. Suganuma and D. Kunii: Kagaku Kogaku Ronbunshu, 3, 12 (1977).

12) Yoshida, T., Y. Kousaka and K. Okuyama: Ind. Eng. Chem., Fundam., 14, 47 (1975).

13) Watanabe, H., T. Takeshita, E. Yamashita and Y. Etou: 37th Kenkyu Danwakai, 75 (1977).

14) Zahradnicek, A. and F. Löffer: Verfahrenstechnik, 11, 229 (1977).

\title{
SULPHUR COMPOUNDS IN COAL TAR AND PETROLEUM THEIR AZEOTROPIC RANGES AND SOME PREDICTED TERNARY AZEOTROPES
}

\author{
ZDZISLAW M. KURTYKA AND ElIZABETH A. KURTYKA \\ University of Guyana, Box 841, Georgetown, Guyana
}

\section{Introduction}

Sulphur occurs in various fractions of petroleum and coal tar in the form of carbon disulphide, alkyl sulphides, thiophene and its derivatives, thionaphthene, etc.

Although the sulphur compounds occur usually in small quantities, their removal by fractional distillation, particularly from the fractions containing aromatics such as benzene, naphthalene, etc., is some-

Received September 5, 1978, Correspondence concerning this article should be addressed to Z. M. Kurtyka, 59 Blaxland Drive, Dandenong, Vic. 3175, Australia. times difficult. For instance, the customary refining methods, if followed by efficient fractional distillation, remove all sulphur compounds from the benzene fraction of light oil except thiophene.

To get some idea about the influence of sulphur compounds on the course of fractional distillation of certain fractions of coal tar and petroleum, it is necessary to know, among other things, their azeotropic ranges and the azeotropes they are able to form.

The purpose of this work was to compute the azeotropic ranges of some sulphur compounds and to predict the existence of some ternary azeotropes con- 